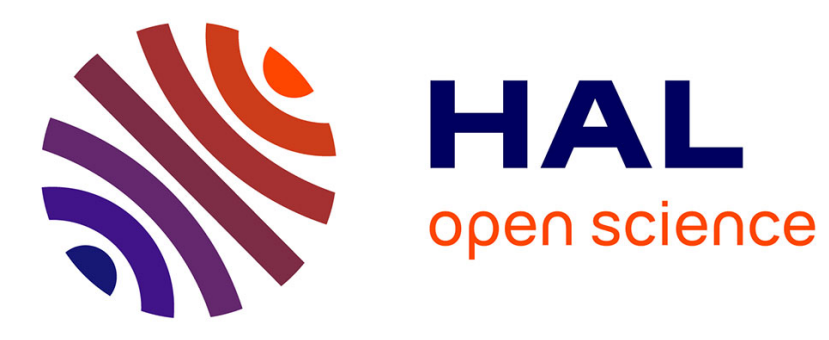

\title{
SAXS investigations of polyethylene modified with methacrylic acid
}

A. Wlochowicz, Cz. Slusarczyk, R. Wycisk, W. Trochimczuk

\section{To cite this version:}

A. Wlochowicz, Cz. Slusarczyk, R. Wycisk, W. Trochimczuk. SAXS investigations of polyethylene modified with methacrylic acid. Journal de Physique IV Proceedings, 1993, 03 (C8), pp.C8-79-C8-82. 10.1051/jp4:1993816 . jpa-00252247

\section{HAL Id: jpa-00252247 https://hal.science/jpa-00252247}

Submitted on 1 Jan 1993

HAL is a multi-disciplinary open access archive for the deposit and dissemination of scientific research documents, whether they are published or not. The documents may come from teaching and research institutions in France or abroad, or from public or private research centers.
L'archive ouverte pluridisciplinaire HAL, est destinée au dépôt et à la diffusion de documents scientifiques de niveau recherche, publiés ou non, émanant des établissements d'enseignement et de recherche français ou étrangers, des laboratoires publics ou privés. 


\title{
SAXS investigations of polyethylene modified with methacrylic acid
}

\author{
A. WŁOCHOWICZ, Cz. ŚLUSARCZYK, R. WYCISK ${ }^{*}$ and W. TROCHIMCZUK* \\ Textile Institute, Technical University of Łódź, Branch in Bielsko-Biata, 43-309 Bielsko-Biata, Willowa 2, \\ Poland \\ * Institute of Organic and Polymer Technology, Technical University of Wroctaw, 50-370 Wrocław, Wybrzeże \\ Wyspiańskiego 27, Poland
}

\begin{abstract}
The polyethylene/poly(methacrylic acid-co-divinylbenzene), PE/poly(MA-co-DVB), interpolymer type carboxylic membranes were studied using small-angle X-ray diffraction (SAXS) in order to deduce the effect of DVB content on their morphology. SAXS curves exhibit a maximum which indicates that the interpolymers have morphology of domain type. Using the standard theory for a two-phase system mean distance between domains and their sizes were calculated from the SAXS data. Analysis of the SAXS curves suggests a fractal behaviour of the internal surface, associated with this two-phase system. Results obtained from SAXS studies are consistent with the general model of these interpolymers revealed by microscopic investigations.
\end{abstract}

\section{Introduction.}

The polyethylene/poly(methacrylic acid-co-divinylbenzene), PE/poly(MA-co-DVB), interpolymer type carboxylic membranes, which are the object of our studies, have been shown to be of heterogeneous type [1]. Preparation details and transport properties of the membranes have been discussed elsewhere [1].

The membranes are made of two polymers: the hydrophobic PE and hydrophilic poly(MA-co-DVB) which, moreover, are known to be immiscible. The morphology of the resulting interpolymer is typical for heterogeneous systems. Microscopic investigations of the membranes containing low DVB contents ( $1-3$ wt\%) revealed that poly(MA-co-DVB) component occurs as spherical domains of dimensions $10-50 \mathrm{~nm}$ dispersed within the PE matrix [1].

The wide-angle X-ray scattering (WAXS) investigations showed that crystallinity of all interpolymers studied is constant and amounts on average to 0.49. This value is the same as for PE film used in interpolymers preparation. It leads to conclusion that poly(MA-co-DVB) neither effect the structure of crystalline regions of PE nor change a weight fraction of this phase in the interpolymers. It suggests that poly(MA-co-DVB) domains are introduced into the amorphous phase of polyethylene.

SAXS analysis was undertaken to characterize the domains and to see whether increasing DVB content would result in increasing crosslinking degree of the domains or for some DVB content a continuous poly(MA-coDVB) network would form that interpenetrated the PE matrix. This could have an important cosequences from the transport viewpoint.

\section{Experimental.}

The PE/poly(MA-co-DVB) interpolymer type carboxylic membranes were prepared by swelling a polyethylene in the form of foil, in mixtures of methacrylic acid, divinylbenzene as a crosslinking agent, and benzoyl peroxide as an initiator at the temperature lower than the dissolution temperature of PE. Then, the swollen foil was heated to interpolimerize the 
comonomers. The comonomers compositions and swelling temperature were chosen so as to obtain interpolymers with ca. 32 wt \% of poly(MA-co-DVB), and DVB content covering the range 0 - 15 wt \%. After the preparation, the samples were treated by turns, with $1 \mathrm{~N} \mathrm{NaOH}$, water, $1 \mathrm{~N} \mathrm{HCl}$ and water, three times succesively.

The interpolymers were transformed into $\mathrm{COOH}$ form by treating with $0.1 \mathrm{~N} \mathrm{HCl}$ for ca. $120 \mathrm{~h}$. Then the samples were washed with distilled water and dried under vacuum.

The small-angle X-ray scattering (SAXS) investigations were carried out by means of a Rigaku-Denki camera in conjuction with $\mathrm{CuK}_{\alpha}$ radiation in an angular range of $2 \theta=0.07^{\circ}$ to $2 \theta=1.01^{\circ}$ with a step $0.01^{\circ}$, and in the range from $2 \theta=1.01^{\circ}$ to $2 \theta=4.01^{\circ}$ with the step of $0.02^{\circ}$. The main beam was defined between the sample and the focal point, at a distance of $330 \mathrm{~mm}$, by two $0.1 \times 10.0 \mathrm{~mm}$ slits and a scattering slit. Between the sample and the detector, $350 \mathrm{~mm}$ apart, there were a receiving slit $0.02 \times 15 \mathrm{~mm}$ and a scattering slit. Monochromatization of the beam was achieved by using a nickel filter and a pulse-height analyser. A scintillation counter was used as a detector.

The experimental data were smoothed and corrected for air scattering, sample absorption, background scattering, and slit height collimation by means of the general computer program FFSAXS-5 elaborated by Vonk [2]. The slit height collimation correction was performed according to Hendricks $[3,4]$.

Before desmearing from the collimation distortions, the diffuse background scattering was subtracted from the scattering curve. It was found that over the scattering angle range of $2 \theta=3.25^{\circ}$ to $2 \theta=4.01^{\circ}$ the intensity of scattered radiation was constant. A mean value of the intensity in this angular range was subtracted as a background. The desmeared curves of scattering were normalized to electron units by comparison to the scattering from a standard (the Lupolen plate supplied by the laboratory of Kratky, Graz).

Samples for transmission electron microscopy (TEM) investigations were stained with uracyl acetate. The microscope used was a Philips EM-301.

\section{Results and discussion.}

It is well known that X-ray scattering at low angle is caused by electron density heterogeneities in the material which is exposed to the radiation. WAXS measurements showed that in the investigated interpolymers one can differentiate three regions with different electron densities, namely: crystalline PE (c), amorphous PE (a), domains of poly(MA-co-DVB) (m) [1].

One can calculated that the electron densities of these phases are: $\rho_{\mathrm{c}}=0.574 \mathrm{~mol} \mathrm{el} / \mathrm{cm}^{3}$, $\rho_{\mathrm{a}}=0.487 \mathrm{~mol} \mathrm{el} / \mathrm{cm}^{3}, \rho_{\mathrm{m}}=0.722 \mathrm{~mol} \mathrm{el} / \mathrm{cm}^{3}$. It indicates that the contrast (i.e. the electron density difference) between crystalline PE and amorphous PE is several times less than that between these phases and the domains of poly(MA-co-DVB). Hence, it is reasonable to assume that the investigated interpolymers are two-phase systems: PE - poly(MA-co-DVB). This assumption is confirmed by comparison of the SAXS pattern for unmodified PE film with SAXS patterns obtained for interpolymers studied.

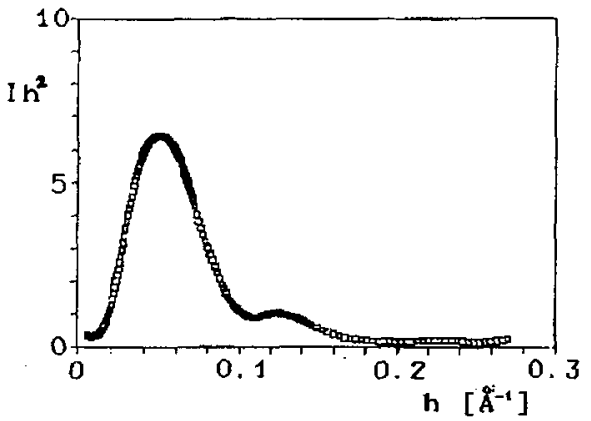

Fig. 1. One dimensional scattering function for polyethylene. $h$ is the value ot the scattering vector, defined as $h=(4 \pi \sin \theta) / \lambda$. Here $\theta$ is half the scattering angle and $\lambda$ the wave length of the $X$ ray.

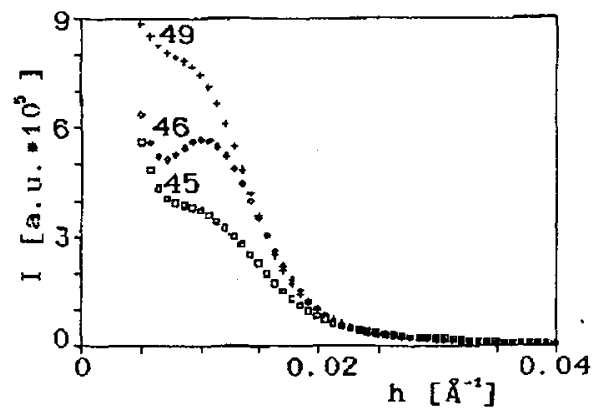

Fig.2. SAXS curves for PE/poly(Ma-co/DVB) interpolymers at different degree of crosslinking. 
The SAXS curve for PE film (Figure 1) exhibits a maximum situated at about $2 \theta=0.7^{\circ}$. This maximum is connected with the lamellar structure of PE. The Bragg's distance corresponding to this maximum is $12.6 \mathrm{~nm}$.

SAXS curves obtained for PE/poly(MA-co-DVB) interpolymers (Fig.2) contain the interference maxima as well but their intensities are much more greater than for PE film and their angular positions are shifted to lower angles as compared with the position of the maximum conditioned by the lamellar structure of PE. It suggests that in this case the maxima indicate the presence of domains and their regular arrangement. Taking into account the interpolymer composition, it may be inferred that these are the domains of poly(MA-co-DVB) dispersed in polyethylene matrix. The mean distances between the domains, obtained from the Bragg's law, are given in Table 1.

In order to estimate the sizes of the poly(MA-co-DVB) domains the correlation length $l_{p}$ was calculated from the slope of the three-dimensional correlation function $\gamma(r)$ at $r=0$, namely

$$
\left(\frac{d \gamma}{d r}\right)_{r \approx 0}=-\frac{1}{I_{p}}
$$

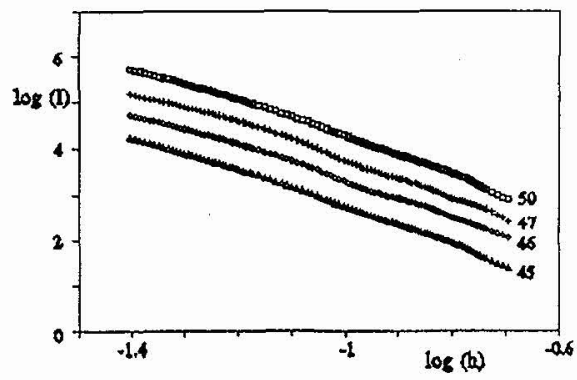

Fig.3. SAXS curves of PE/poly(MA-coDVB)interpolymers on double logarithmic scales, which indicate on fractal behaviour. The successive curves are displaced to avoid overlapping. The numbers on the curves are to identify the sample.

Using the $1_{p}$ and the volume fraction of the phases, $w_{1}$ and $w_{2}$, the values of $l_{1}$, the transversal length in PE and $l_{2}$, the transversal length in poly(MA-Co-DVB) domains were found from equation $l_{p}=l_{1} w_{2}=l_{2} w_{1}$. Results are presented in Table 1 .

The largest value of the transversal length $l_{2}$ is obtained for sample containing $3 \mathrm{wt} \%$ of DVB. Then with the increasing content of DVB, dimensions of domains decrease.

Figure 3 reveals a linear behaviour for $\ln (I)$ versus $\ln (h)$ in the middle part of the SAXS curves. Such behaviour is typical for fractals described in literature [5]. The main parameters describing this fractal behaviour are: the fractal dimension D and the fractal region (in length units). The fractal dimension characterizes the degree of ramification of the phase boundary surface and it can be obtained from the slope (p) of the graph of $\ln (\mathrm{I}) v s . \ln (\mathrm{h})$ by the expression $\mathrm{D}=6$-p. The fractal region is calculated from a region of regression so limited as to obtain an optimal straight line. These parameters obtained for investigated samples are given in Table 2.

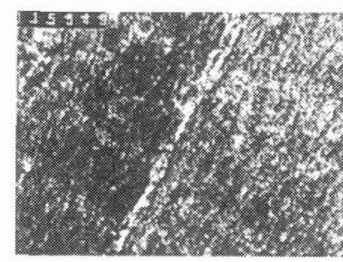

a

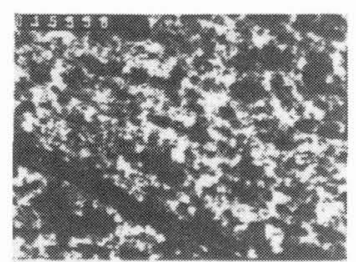

b
Fig.4. TEM micrographs of thin section of the interpolymer stained with uracyl acetate. a) sample 47; b) sample 50. Magnification 5900x.
The increase in DVB content firstly causes the smoothness of the separation surface between PE and poly(MA-co-DVB) domains and next the ramification of this surface. The separation surface is most smooth within the sample 47 containing 3 $w t \%$ of DVB, i.e. for the sample which exhibits the largest value of $\mathrm{I}_{2}$. Changes of the fractal dimension of the separation surface very well reflect the changes of the sizes of poly(MA-co-DVB) domains. It should be noticed as well that the maximum length of the fractal region sufficiently well corresponds to the transversal length $l_{2}$ for all interpolymers studied.

Summarizing the SAXS data it should be stated that the effect of the DVB content on the microphase pattern in $\mathrm{PE} /$ poly(MA-co-DVB) interpolymers is 
pattern in PE/poly(MA-co-DVB) interpolymers is complex. The interpolymer without DVB (sample 45) has very heterogeneous morphology characterized by poor definite domains of poly(MA-co-DVB) and the rough boundary between PE and domains. The membranes containing low DVB contents (1-3 wt\%) have well determined domains characterized by the smooth boundary. However for high DVB content (15 wt\% - sample 50) the sizes of domains again decrease and the surface of phase boundary becomes more rough. But for all interpolymers studied no disappearance of the domain structure with increasing DVB content takes place. The ramification of the phase boundary surface for high content of DVB is probably connected with the cluster formation revealed lately by microscopic investigations [6]. It has been observed that for the sample containing $15 \mathrm{wt} \%$ of DVB small poly(MA-co-DVB) domains form clusters of dimensions 100-300 nm (Figure 4).

A more complete interpretation of the effect of DVB content on the structure of PE/poly(MA-co-DVB) interpolymers requires futher experiments using SAXS and electron microscopy on more samples.

Table 1. SAXS data for PE/poly(MA-co-DVB) interpolymers of different DVB content

\begin{tabular}{|c|c|c|c|c|c|c|c|}
\hline Sample & $\begin{array}{l}\text { Poly(MA-co- } \\
\text { DVB) content }\end{array}$ & DVB content & $\begin{array}{c}\text { Volume } \\
\text { fraction } \\
\text { of PE } \\
w_{1} \\
\end{array}$ & $\begin{array}{l}\text { Bragg } \\
\text { distance }\end{array}$ & $\begin{array}{l}\text { Correlation } \\
\text { length } L_{p}\end{array}$ & $\begin{array}{l}\text { Transversal } \\
\text { length } \\
l_{1}\end{array}$ & $\begin{array}{l}\text { Transversal } \\
\text { length } \\
1_{2}\end{array}$ \\
\hline- & [wt\%] & [wt\%] & - & [nm] & [nm] & [nm] & [nm] \\
\hline 45 & 31.8 & 0.0 & 0.757 & 44.1 & 9.7 & 39.8 & 12.8 \\
\hline 46 & 32.3 & 1.6 & 0.753 & 49.0 & 12.7 & 52.0 & 16.8 \\
\hline 47 & 31.2 & 3.0 & 0.762 & 55.1 & 13.0 & 53.3 & 17.1 \\
\hline 48 & 31.5 & 6.7 & 0.760 & 49.0 & 11.4 & 46.7 & 15.0 \\
\hline 49 & 31.9 & 10.5 & 0.756 & 46.4 & 10.0 & 41.0 & 13.2 \\
\hline 50 & 32.2 & 15.4 & 0.754 & 55.1 & 10.2 & 41.8 & 13.5 \\
\hline
\end{tabular}

Table 2. The slopes $p$ of the fractal plots of Fig.2. are given with the fractal dimension D =6-p. The limiting length values of the fractal region are obtained from $2 \pi / \mathrm{h}$ (Bragg's law)

\begin{tabular}{|c|c|c|c|}
\hline Sample & Slope $p$ & Fractal dimension & D \\
\hline 45 & -3.85 & 2.15 & $\begin{array}{c}\text { Fractal region } \\
{[\mathrm{nm}]}\end{array}$ \\
\hline 46 & -3.87 & 2.13 & $18.0-5.5$ \\
\hline 47 & -3.99 & 2.01 & $17.5-6.4$ \\
\hline 48 & -3.90 & 2.10 & $19.2-9.2$ \\
\hline 49 & -3.82 & 2.14 & $16.0-4.0$ \\
\hline 50 & -3.82 & 2.18 & $16.0-7.8$ \\
\hline
\end{tabular}

\section{References.}

[1] WYCISK R.,TROCHIMCZUK W., J. Appl. Polym. Sci. 43 (1991) 1727.

[2] VONK C.G., J. Appl. Cryst. 8 (1975) 340.

[3] HENDRICKS R.W., SCHMIDT P., Acta Physica Austriaca 26 (1967) 97.

[4] HENDRICKS R.W., SCHMIDT P., Acta Physica Austriaca 37 (1970) 20.

[5] SCHMIDT P., J. Appl. Cryst. 24 (1991) 414.

[6] WYCISK R., PhD Thesis, Technical University of Wroclaw, 1993. 